\title{
RESTRICCIONES ECONÓMICAS EN LA DECISIÓN DE CONTINUAR ESTUDIOS SUPERIORES TÉCNICOS O PROFESIONALES
}

\section{Introducción}

La educación superior tiene una reconocida alta rentabilidad social y privada, aspecto que ha contribuido a generar el fenómeno conocido como "masificación de la educación superior". Esto, sumado al hecho de que sus beneficios sean principalmente internalizados por los estudiantes, parece haber impulsado a muchos países, incluso algunos con esquemas rigurosamente estatales, a abrirse a soluciones de financiamiento privado o mixto (Brunner et al., 2005; Leiva, 2005; Sapelli, 2003; Palacios, 2001; Arellano y Braun, 1999; Tilak, 1993; Patrinos, 1990; Stone, 1990).

Entre los países menos desarrollados, Chile ha sido pionero en la masificación de la educación superior. El interés por continuar estudios superiores ha aumentado como consecuencia de diversos factores, entre otros, el avance de la educación media en el país y las expectativas de las familias que ven en ellos un vehículo privilegiado de ascenso social (PNUD, 2006). Sin embargo, las diferentes oportunidades -derivadas de distintas situaciones económicas- parecen haber sido determinantes en la admisión a estos estudios. Sólo un porcentaje pequeño de las familias chilenas puede financiar la educación superior de sus hijos, por lo que las ayudas estudiantiles -en forma de becas y créditos- son fundamentales para generar oportunidades en los sectores más desprotegidos (Cáceres y Chávez, 1995; Leiva, 2003; Bernasconi, 2006; Larrañaga, 2006; PNUD, 2006).

Históricamente, el financiamiento público de la educación superior en Chile se ha concentrado en las universidades tradicionales, que ofrecen principalmente carreras profesionales. Por su parte, 
instituciones que imparten carreras técnicas, como los centros de formación técnica (CFT), han debido recurrir casi completamente a los aranceles y matrículas para financiar sus costos (Larrañaga, 2006). Como resultado, ha quedado en desventaja respecto de la formación profesional, porque un importante grupo de estudiantes meritorios no puedan continuar estudios superiores por las restricciones económicas que enfrentan (PNUD, 2006).

La estrechez de recursos de los jóvenes se refleja en la Encuesta de Caracterización Socioeconómica Nacional (CASEN). En 2003, 468.305 jóvenes ${ }^{1}$ cursaban carreras profesionales, 157.308 cursaban carreras técnicas y 1.249.151 jóvenes, ya egresados de educación media, no se matricularon en ningún tipo de institución superior. Esta distribución se asocia al ingreso familiar, dado que el 67,20\% de quienes estaban cursando carreras profesionales y el 52,56\% de los que cursaban carreras técnicas pertenecían al 40\% de los egresados de enseñanza media de mayores ingresos per cápita (tabla 1$)^{2}$.

Tabla 1: Composición de cada grupo por quintil

\begin{tabular}{l|c|c|c|c|c|c}
\hline \multicolumn{1}{c|}{ Grupo } & Quintil I & Quintil II & Quintil III & Quintil IV & Quintil V & Total \\
\hline Profesional & $8,83 \%$ & $9,34 \%$ & $14,64 \%$ & $23,00 \%$ & $44,20 \%$ & 468.305 \\
\hline Técnico & $10,68 \%$ & $16,13 \%$ & $20,63 \%$ & $24,10 \%$ & $28,46 \%$ & 157.308 \\
\hline Trabajo & $25,39 \%$ & $24,52 \%$ & $21,97 \%$ & $18,42 \%$ & $9,71 \%$ & 1.249 .151 \\
\hline
\end{tabular}

Fuente: CASEN, 2003.

A su vez, entre los jóvenes, el ingreso per cápita promedio del primer quintil era \$20.296; el del segundo quintil \$50.360; el del tercero $\$ 78.432$; el del cuarto $\$ 125.279$ y el del quinto $\$ 429.969$ (CASEN, 2003). Así, más del 60\% de quienes se enfrentaron a la decisión de estudiar tenían un ingreso per cápita menor a los $\$ 100.000$, aspecto que limitaba enormemente su elección ${ }^{3}$.

1 Para efectos del presente estudio se consideraron jóvenes entre 17 y 30 años que tenían al menos la enseñanza media completa.

2 Todas la información estadística del presente estudio se encuentra disponible en el sitio del CSE: www.cse.cl

3 Base estadística "Indicadores, Números y Datos sobre Instituciones y Carreras de Educación Superior" (INDICES) del año 2005, elaborada por el Consejo Superior de Educación (CSE). 
Las restricciones en las elecciones y la asignación de los recursos fiscales explican, en parte, la estructura de la educación superior, muy distinta de la que prevalece en los países desarrollados. En Chile, la educación profesional representa una fracción significativamente mayor de los estudiantes postsecundarios, hecho que se ha acrecentado en los últimos años (Leiva, 2003; Dittborn y Aedo, 2005; Larrañaga, 2006).

Los estudios sobre decisión postsecundaria, principalmente para los Estados Unidos, muestran que existe una gran variedad de factores que influyen al momento de escoger un programa, entre otros: el costo de la carrera, la ayuda financiera, la ubicación geográfica, las exigencias del proceso de admisión, la calidad académica, la reputación, la amplitud de las áreas del conocimiento que se ofrecen, el tamaño, la atmósfera social. Estos estudios, sin embargo, no revelan un claro patrón respecto de la importancia que los postulantes otorgan a cada uno de estos factores (Hossler et al., 1989; Galotti y Mark, 1994).

En Chile sólo se han estudiado grupos muy específicos. De acuerdo con entrevistas realizadas por Razcynski y Canales (2000), los estudiantes chilenos con altos puntajes en las pruebas de admisión tienden a decidir en relación con la reputación o calidad académica de la carrera o institución. Por su parte, los de menor rendimiento académico atienden a una serie más amplia de factores, entre los que puede encontrarse o no la calidad. Los alumnos provenientes de familias de mayores ingresos tienden también a tener más acceso y a demandar más información para tomar sus decisiones (Lemaitre y Raczynski, 2001). Según Bernasconi (1998), tanto factores sociales como personales estructuran las elecciones de los estudiantes; entre los primeros, las aspiraciones puestas en la educación superior como vehículo de movilidad social y como promesa de mejor calidad de vida. Lo cierto es que la racionalidad de las decisiones de los individuos surge sólo después de superar las restricciones que enfrentan.

En general, los estudios chilenos se han centrado en las políticas de financiamiento y la rentabilidad de los distintos sectores 
de la educación, sin preocuparse mayormente del grado en que estas variables afectan la decisión de continuar estudios superiores. Esto obedece a la carencia de fuentes de información que liguen tales decisiones con datos institucionales desagregados. El propósito de este artículo es relacionar las distintas fuentes de datos y, a partir de ello, determinar regularidades en el comportamiento de los jóvenes que, al terminar la educación media, se enfrentan a la decisión de estudiar una carrera profesional o técnica, o de no seguir estudiando.

Para ello, usamos tres fuentes de datos: a) la Encuesta CASEN, elaborada por MIDEPLAN para 2003, realizada en hogares y representativa en el plano nacional; b) la base de datos "Indicadores, Números y Datos sobre Instituciones y Carreras de Educación Superior" (INDICES) 2005, que reúne información recolectada por el Consejo Superior de Educación (CSE), y c) la base de datos de la Prueba de Aptitud Académica (PAA) de 2002, del Departamento de Evaluación, Medición y Registro Educacional (DEMRE).

Este documento consta de tres secciones. La primera describe el modelo de decisión de elección y la metodología para crear la imputación de las variables de interés, la segunda presenta los resultados de la estimación del modelo de elección y la tercera concluye con los principales resultados.

\section{Modelo y metodología}

\section{El modelo}

Al terminar su escolaridad obligatoria de 12 años, los estudiantes se enfrentan a distintas opciones: seguir una carrera profesional (opción 1), una carrera técnica superior (opción 2), o, simplemente, no seguir estudiando, alternativa que asimilamos más directamente al trabajo

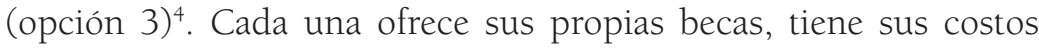

4 Para ver la comparación entre universidades (opción 1), institutos profesionales (IP) y CFT (opción 2) y no continuar estudiando (opción 3), véase Hernández, 2007. 
directos, proyecta distintos ingresos, requiere diferentes esfuerzos y habilidades, y demanda diversas capacidades.

Las decisiones de escolaridad se enmarcan en el contexto de la "teoría de capital humano". Aunque el valor económico de la educación ha sido considerado en la literatura desde los economistas clásicos, la introducción de esta teoría considera la educación como una inversión (Schultz, 1960; Becker, 1964; Mincer, 1974). Se la concibe como parte del acervo de capital humano y un instrumento que aumenta la productividad y los salarios.

Siguiendo a Becker (1964), la persona tiene que elegir entre caminos que le otorgarán distintos flujos de beneficios. Si decide no continuar estudios superiores, parte de los ingresos son percibidos inmediatamente, pero en el futuro no llegarán a ser muy altos; si decide seguirlos, tendrá un flujo negativo los primeros años (costos directos más costo de oportunidad) para luego comenzar a recibir ingresos que superarán los de la otra alternativa. En general, invertir en educación superior se justifica si el valor presente de los beneficios es, al menos, tan grande como el de los costos.

De la teoría de capital humano se derivan predicciones claras: a) la educación es, predominantemente, una actividad realizada cuando se es joven; b) disminuye el atractivo de la educación superior si los costos directos aumentan y menor es la diferencia entre los ingresos de sus titulados y los de graduados de enseñanza media, y c) siendo el capital humano no colateralizable, las restricciones de liquidez limitan el acceso a la educación.

La decisión de la educación postsecundaria depende entonces de los beneficios brutos de cada opción (valor presente del flujo de ingresos generado), de los costos de cada una de ellas -en la medida en que hay costos y retornos diferentes- y de las limitaciones en la admisión (Becker, 1964). Por su parte, las restricciones económicas están determinadas por el costo de la carrera, las becas y créditos, y la situación económica del individuo. Como señalamos, estas 
restricciones existen por la imposibilidad de colateralizar al capital humano en el mercado del crédito, es decir, éste no se puede hipotecar. Sin embargo, no sólo existen estas restricciones; muchos programas de educación superior, particularmente los de mayor prestigio, aplican restricciones no económicas por su capacidad limitada o, más correctamente, por la dificultad de ajustar su oferta en el corto plazo. Específicamente, la selección no económica se basa en el rendimiento académico o aptitud, medido en 2002 a través del puntaje de la PAA y de las notas en la educación media. Estas restricciones permiten que, en el margen, existan diferencias de retornos netos de distintas opciones seguidas.

En consecuencia, el modelo a estimar es:

$$
\text { Elección }_{\mathrm{i}}=\mathrm{f}\left(\mathrm{Y}_{\mathrm{i}} ; \mathrm{RL}_{\mathrm{i}} ; \mathrm{RC}_{\mathrm{i}}\right)
$$

en el cual Elección se refiere a la opción del estudio postsecundario (profesional o técnico) o a no estudiar; Y representa los ingresos generados por las tres distintas elecciones; RL las restricciones de liquidez que consideran los costos directos e indirectos, las becas y los créditos esperados de seguir cada opción de estudio y la situación económica, y $R C$ las restricciones de capacidad de los programas de educación superior que seleccionan por rendimiento académico.

\section{Metodología}

La información requerida para estimar (1) no está disponible directamente. La encuesta CASEN contiene la elección de los individuos, pero no el rendimiento académico ni los ingresos, becas y créditos esperados en cada opción de estudio. Además, no hay forma de identificar las situaciones caso a caso. Por ello, mostramos en lo que sigue la forma en que imputamos las variables proxy, en las cuales lo central es el uso de predicciones a partir de fuentes de datos complementarias. Al concluir todas las predicciones, será posible estimar (1), logrando explicar las decisiones de los jóvenes con los ingresos esperados en el futuro y las restricciones de liquidez y de capacidad. Esto significa determinar la importancia de cada una 
de estas variables en cada opción y cuantificar sus efectos sobre las decisiones de los estudiantes.

\section{a) Rendimiento académico}

El rendimiento académico de los alumnos que terminan la enseñanza media -que se usa como proxy del que pudieran mostrar en la educación superior- es una combinación del puntaje de la PAA y las notas. Nosotros consideramos ponderaciones de $80 \%$ para el puntaje promedio entre lenguaje y matemáticas y de $20 \%$ para el puntaje asociado a las notas del alumno, basado en los requisitos usados por las universidades.

La información de las personas que rindieron la PAA en 2002 proviene del DEMRE (2002). Este indicador, sin embargo, debe ser imputado a los alumnos egresados de la enseñanza media. Con este propósito realizamos la siguiente regresión lineal:

$$
R A_{i}=f\left(V S D_{i} ; T_{i} ; R_{i}\right)
$$

en la que RA es el rendimiento académico; VSD un vector de variables sociodemográficas que incluye sexo, edad, tamaño familiar y educación de los padres; TC un vector que contiene el tipo de colegio - particular pagado, particular subvencionado o municipal- y $R$ un vector con dummies ${ }^{5}$ para las regiones. Los resultados más relevantes de la regresión se muestran en la tabla 2.

Tabla 2: Regresión de rendimiento académico

\begin{tabular}{l|c|c}
\hline Variable & Coef & $(\mathrm{z})$ \\
\hline Sexo & $11,9^{*}$ & $(26,6)$ \\
\hline Colegio privado & $39,4^{*}$ & $(54,8)$ \\
\hline Colegio municipal & $-13,9^{*}$ & $(-27,2)$ \\
\hline Edad & $-0,1^{*}$ & $(-2,0)$ \\
\hline Tamaño de la familia & $-2,6^{*}$ & $(-20,2)$ \\
\hline Dummies regionales & significativas & \\
\hline Educación del padre & significativa & \\
\hline
\end{tabular}

5 Variables binarias, de resultado 0 o 1 . 


\begin{tabular}{l|c|c}
\hline Educación de la madre & significativa & \\
\hline Constante & $466,4^{*}$ & $(223,5)$ \\
\hline $\mathrm{N}$ & 178.652 & \\
\hline
\end{tabular}

Nota: Los coeficientes significativos al 5\% se indican con *.

Los resultados más llamativos se relacionan con la sensibilidad del puntaje ante cambios en el tipo de colegio, la educación de los padres y las diferencias entre hombres y mujeres. En el caso de los colegios, los puntajes esperados para quienes provengan de particulares pagados son casi 40 puntos mayores a los esperados para los de colegios particulares subvencionados, los que, a su vez, son mayores en 14 puntos a los de colegios municipales. Estas enormes diferencias determinan las probabilidades de la mayoría de los alumnos, puesto que el 50\% de ellos está en colegios municipales.

La educación de los padres tiene un impacto importante en los puntajes estimados, con diferencias superiores a los 50 puntos en algunos casos, como cuando se trata de un padre con educación universitaria completa en relación con otro que no tenga estudios. El efecto de la educación de las madres es estadísticamente mayor al de la educación de los padres, al comparar dos niveles equivalentes. Asimismo, el puntaje esperado aumenta conforme aumenta el nivel educacional de la madre, mientras que en el caso del padre se da en todos los niveles, menos al pasar de no tener estudios a haber llegado a la educación básica, en el cual se produce una disminución ${ }^{6}$ (para más detalle, véase Hernández, 2007). Finalmente, los resultados esperados son 11,9 puntos mayores en hombres $(S E X O=1)$ que en mujeres.

A partir de los resultados de la tabla 2, imputamos el rendimiento académico a las personas en la CASEN 2003 ${ }^{7}$. Desafortunadamente, la información sobre el tipo de colegio de los egresados de enseñanza

6 Esto podría deberse al fenómeno de sobreselección social o hiperselección (Bourdieu, 2000; Bourdieu y Passeron, 2003; García de León, 2005).

7 Debido a la escasez de información, si los jóvenes no viven con los padres se reemplaza la educación de éstos por la del jefe de núcleo y cónyuge. En caso de que el joven sea el jefe o cónyuge, se ocupa sólo una para reemplazar ambas. 
media no se encuentra disponible. Como esta variable es muy determinante del rendimiento, se procedió a estimar la probabilidad de haber asistido a cada tipo de colegio con la misma información de la CASEN 2003, usando el siguiente modelo multinomial probit ${ }^{8}$ :

$$
\mathrm{TC}_{\mathrm{i}}=\mathrm{f}\left(\mathrm{SEXO}_{\mathrm{i}} ; \mathrm{DECIL}_{\mathrm{i}} ; \mathrm{R}_{\mathrm{i}}\right)
$$

en el cual TC es el tipo de colegio (municipal, particular subvencionado o particular privado); SEXO es una dummy con valor 1 si es hombre, y DECIL es un vector con dummies para cada decil (decil 10 es base). La tabla 3 contiene los efectos marginales de cada una de las variables que explican el tipo de colegio del alumno.

Tabla 3: Efectos marginales en la probabilidad de venir de cada tipo de colegio

\begin{tabular}{l|c|c|c|c|c|c}
\hline \multicolumn{3}{|c|}{ Municipal } & \multicolumn{2}{c|}{ Particular subvencionado } & \multicolumn{2}{c}{ Part. pagado } \\
\hline Var & dy/dx & $(\mathrm{Z})$ & dy/dx & $(\mathrm{Z})$ & dy/dx & $(\mathrm{Z})$ \\
\hline SEXO & $0,009^{*}$ & $(16,0)$ & $-0,006^{*}$ & $(-11,8)$ & $-0,003^{*}$ & $(-11,5)$ \\
\hline D1 & $0,394^{*}$ & $(483,5)$ & $-0,318^{*}$ & $(-396,9)$ & $-0,075^{*}$ & $(-390,1)$ \\
\hline D2 & $0,409^{*}$ & $(529,0)$ & $-0,333^{*}$ & $(-437,5)$ & $-0,076^{*}$ & $(-393,7)$ \\
\hline D3 & $0,361^{*}$ & $(406,4)$ & $-0,285^{*}$ & $(-325,6)$ & $-0,076^{*}$ & $(-390,1)$ \\
\hline D4 & $0,336^{*}$ & $(357,2)$ & $-0,262^{*}$ & $(-281,9)$ & $-0,075^{*}$ & $(-383,1)$ \\
\hline D5 & $0,312^{*}$ & $(314,0)$ & $-0,237^{*}$ & $(-241,8)$ & $-0,075^{*}$ & $(-385,5)$ \\
\hline D6 & $0,285^{*}$ & $(272,4)$ & $-0,212^{*}$ & $(-205,6)$ & $-0,072^{*}$ & $(-376,0)$ \\
\hline D7 & $0,223^{*}$ & $(193,1)$ & $-0,153^{*}$ & $(-133,8)$ & $-0,070^{*}$ & $(-370,2)$ \\
\hline D8 & $0,159^{*}$ & $(127,8)$ & $-0,093^{*}$ & $(-75,8)$ & $-0,066^{*}$ & $(-360,3)$ \\
\hline D9 & $0,087^{*}$ & $(65,3)$ & $-0,032^{*}$ & $(-24,7)$ & $-0,055^{*}$ & $(-330,8)$ \\
\hline Provincias & significativas & & significativas & & significativas & \\
\hline R.M. & $-0,115^{*}$ & $(-71,1)$ & $0,058^{*}$ & $(37,2)$ & $0,057^{*}$ & $(62,1)$ \\
\hline N & 3.809 .754 & \multicolumn{5}{|l}{} \\
\hline
\end{tabular}

Nota: Los coeficientes significativos al $5 \%$ se indican con *

Los resultados muestran que la probabilidad de estar en un colegio municipal disminuye a medida que aumentan los ingresos, mientras que la de provenir de alguno de los otros dos tipos de colegios aumenta. Los cambios en la probabilidad al pasar de un decil al siguiente son bajos en los colegios particulares privados hasta el

8 Se trata de una regresión en la cual la variable dependiente (TC) toma valores categóricos. En este caso responde a tres categorías. 
último decil, donde aumentan considerablemente (5,5\%), prueba de que se trata de una educación de elite económica concentrada en el décimo decil. En cuanto a los resultados por regiones, destaca el hecho de que los alumnos de la Región Metropolitana tienen mayor probabilidad de provenir de colegios subvencionados y privados, lo que se debe a una mayor proporción de colegios municipales en otras regiones.

A partir de las probabilidades de provenir de cada tipo de colegio, proyectamos el rendimiento académico estimado reemplazando las probabilidades en la regresión (2). Los resultados indican que existe una tendencia a aumentar el puntaje a medida que aumentan los ingresos en las tres alternativas y que en todos los quintiles el promedio del rendimiento académico estimado de quienes están en carreras profesionales es superior a aquellos que cursan carreras técnicas, y éste es mayor al de quienes no continúan estudios superiores.

\section{b) Ingresos esperados}

Para estimar los ingresos esperados que provendrían de cada opción, considerando a todos los que tienen 12 años de escolaridad o se titularon en uno de los dos tipos de educación superior analizados (se excluyen alumnos actuales), seguimos a Heckman (1979) y estimamos una función de salarios corregida por el posible sesgo de selección que genera el hecho de que los empleados no son una muestra representativa de la población. Ello se sintetiza en la siguiente regresión:

$$
\operatorname{In}\left(\mathrm{W}_{\mathrm{i}}\right)=\mathrm{f}\left(\mathrm{SEXO}_{\mathrm{i}} ; \mathrm{EDAD}_{\mathrm{i}} ; \mathrm{R}_{\mathrm{i}} ; \mathrm{EC}_{\mathrm{i}} ; \lambda_{\mathrm{i}}\right),
$$

según la cual $W$ es el ingreso mensual futuro; $E C$ es un vector de dummies para cada nivel educacional del cónyuge (sin estudios es base); y $\lambda$ es el inverso de la razón de Mills que controla el sesgo de selección.

El hecho de que el modelo se base en personas que ya decidieron y que a partir de ellos se desee predecir ingresos de 
quienes actualmente deben decidir, justifica la inclusión de variables que aporten información de su pasado. Como no se conoce este tipo de información, se utilizó la educación del cónyuge, lo que puede capturar algo del impacto de la condición socioeconómica pasada. Al momento de imputar esta información en los jóvenes que deben decidir, si éstos no tienen cónyuge, se utilizó la educación del jefe del núcleo familiar. Los resultados de la estimación en las tres opciones se presentan en la tabla 4.

Tabla 4: Modelo de Heckman de ingresos esperados

\begin{tabular}{l|c|c|c|c|c|c}
\hline & \multicolumn{2}{|c|}{ Profesional } & \multicolumn{2}{c|}{ Técnico } & \multicolumn{2}{c}{ Trabajo } \\
\hline Variable & Coef & $(z)$ & Coef & $(z)$ & Coef & $(z)$ \\
\hline EDAD & $0,08^{*}$ & $(103,05)$ & $0,06^{*}$ & $(69,6)$ & 0,07 & $(129,2)^{*}$ \\
\hline EDAD $^{2}$ & $-0,00^{*}$ & $(-98,42)$ & $-0,00^{*}$ & $(-55,4)$ & $-0,00$ & $(-120,7)^{*}$ \\
\hline SEXO & $0,67^{*}$ & $(116,94)$ & $0,40^{*}$ & $(39,1)$ & 0,58 & $(117,2)^{*}$ \\
\hline Región & signif. & & signif. & & signif. & \\
\hline Escolaridad cónyuge & signif. & & signif. & & signifs. & \\
\hline Constante & $10,44^{*}$ & $(394,10)$ & $11,20^{*}$ & $(404,3)$ & 10,00 & $(564,4)^{*}$ \\
\hline$\lambda$ & $0,83^{*}$ & $(25,64)$ & $-0,10^{*}$ & $(-3,05)$ & 0,13 & $(12,61)^{*}$ \\
\hline $\mathrm{N}$ & 635.706 & & 377.091 & & 1.946 .254 & \\
\hline
\end{tabular}

Nota: Los coeficientes significativos al $5 \%$ se indican con *

En todos los casos se observa que los mayores ingresos esperados son para hombres, siendo la diferencia de alrededor de un 60\%. La diferencia en los ingresos al aumentar un año de edad es positiva hasta los 55 años en profesionales, 61 años para técnicos superiores y 58 años en personas con estudios secundarios completos; a partir de ese nivel, un año más disminuye los ingresos esperados ${ }^{9}$.

Siguiendo con el proceso de construcción de la base de datos que permita estimar (1), basándose en la regresión (4), imputamos los salarios a cada persona, proyectados a la edad de 30 años, en función de la alternativa escogida.

9 Esto se obtiene a partir de la regresión (4), calculando $\frac{\mathrm{dW}}{\mathrm{dEDAD}}=\mathrm{W}\left(\delta_{2}+2 \delta_{3} \mathrm{EDAD}\right)=0$. 


\section{c) Becas y créditos esperados}

La información disponible incluye cuatro grupos: a) la Beca Presidente de la República, un aporte monetario de libre disposición equivalente a 1,24 UTM por diez meses (marzo a diciembre); b) la Beca de Matrícula o Arancel (ex MINEDUC), destinada a financiar parcial o totalmente el arancel anual de la carrera; c) todas las otras becas entregadas por el Estado -Juan Gómez Millas, para Estudiantes Destacados que Ingresan a Pedagogía, para Estudiantes Hijos/Hijas de Profesionales de la Educación y Nuevo Milenio, entre otras-, y d) todas aquellas becas que no son otorgadas por el Estado.

La información de créditos también incluye cuatro grupos: a) el Crédito Universitario o Fondo Solidario, que se otorga en UTM con tasa de interés anual del 2\% y se empieza a cancelar después de dos años de haber egresado, pagando anualmente una suma equivalente al 5\% del total de ingresos que haya obtenido el año anterior; b) el Crédito Corfo, una línea de financiamiento abierta en los bancos por la Corporación de Fomento de la Producción para que otorguen créditos a estudiantes chilenos (provenientes principalmente de familias de ingresos medios), con el fin de facilitarles el pago de estudios superiores de pregrado; c) todos aquellos créditos otorgados por el establecimiento, y d) todos los créditos que no caben en los grupos anteriores.

El método utilizado para estimar los montos esperados de becas y créditos consistió en multiplicar la probabilidad de obtener alguno de ellos por su monto estimado. Tanto para la estimación de la probabilidad como del monto se utilizaron como variables explicativas el rendimiento académico, el sexo, la región, la edad y el quintil de ingreso per cápita familiar.

Aunque, de acuerdo con sus bases, los criterios de asignación que se esperarían en la entrega de ayudas estudiantiles fueran la necesidad económica y el rendimiento académico, sorprende lo ineficaces que resultaron como predictores de las regresiones de todas las becas y créditos (se realizaron regresiones para estimar la 
probabilidad y el monto en cada uno de los cuatro tipos de becas y créditos $)^{10}$. Este tema es crítico, porque sugiere que las personas que postulan a este tipo de beneficios no son representativas de la población y los criterios de asignación se dan sólo entre postulantes; ello redundaría en que una enorme cantidad de personas, que pudiese merecer o necesitar las ayudas estudiantiles, se quedaría fuera del proceso.

Finalmente, tanto con becas como con créditos, calculamos un monto total sumando los cuatro tipos y truncándolos por arriba con el costo de la carrera, utilizando para ello la base de datos INDICES 2005. El fundamento de usar un solo monto agregado para becas y uno para créditos, es que, en general, cuando una persona decide, no toma en cuenta los valores por separado sino que su total, independiente de cuál sea su origen. Las personas que no continúan estudios superiores tienen una expectativa de mayores ayudas estudiantiles que quienes sí lo hacen, lo que sugiere que los montos totales que se están entregando no son suficientes para resolver las restricciones de liquidez. Esto es, aun cuando una persona espere recibir uno de los mayores montos, no le alcanzará para pagar el arancel, dada su mala situación económica.

Una vez terminada la construcción de la base de datos, es posible evaluar y cuantificar ahora los efectos de los principales determinantes de la elección por carreras profesionales, técnicas o por no continuar estudios superiores.

\section{Determinantes de la elección}

La decisión que toman los egresados de enseñanza media entre seguir una carrera profesional, técnica y no continuar estudios

10 Los resultados detallados no se presentan por ser muy extensos (cuatro tipos de becas, cuatro de créditos y dos regresiones para cada una de ellas en cada una de las tres opciones). En Hernández (2007) se puede encontrar un análisis que sigue la misma lógica al comparar la decisión entre ingresar a la educación universitaria y a la educación superior no universitaria, obteniendo resultados similares en lo referente a las ayudas estudiantiles. 
superiores, considera la maximización de los ingresos futuros, sujeto a sus restricciones de liquidez y a las limitaciones de capacidad de los programas de educación superior. Para estimar el efecto de los distintos determinantes de la elección, se optó por el siguiente modelo multinomial probit:

$$
\begin{aligned}
\text { ELECCIÓN }_{\mathrm{i}}= & \alpha_{1} \mathrm{RA}_{\mathrm{i}}+\alpha_{2} \mathrm{RA}_{\mathrm{i}}{ }^{2}+\alpha_{3} \mathrm{Wl}_{\mathrm{i}}+\alpha_{4} \mathrm{~W}_{\mathrm{i}}+\alpha_{5} \mathrm{~W}_{\mathrm{i}}+\alpha_{6} \mathrm{Bl}_{\mathrm{i}}+\alpha_{7} \mathrm{~B}_{\mathrm{i}}+\alpha_{8} \mathrm{Cl}_{\mathrm{i}}+ \\
& \alpha_{9} \mathrm{C}_{\mathrm{i}}+\alpha_{10} \mathrm{Q}_{\mathrm{i}}+\ldots+\alpha_{13} \mathrm{Q}_{\mathrm{i}}+\alpha_{14}+\mathrm{J}
\end{aligned}
$$

en el que ELECCIÓN considera las tres alternativas señaladas; los $\alpha_{k}$ son los parámetros a estimar; $R A$ es el rendimiento académico estimado; W1, W2 y W3 son los ingresos mensuales esperados a los 30 años de edad, cuando se titulan de carreras profesionales, técnicas o cuando no continúan estudiando, respectivamente; B1 y B2 son las becas esperadas en carreras profesionales o técnicas, respectivamente; $\mathrm{C} 1$ y $\mathrm{C} 2$ son los créditos esperados en carreras profesionales o técnicas, respectivamente; Qj es una dummy para el quintil j (quintil 5 es base); y $\vartheta$ es un término de error estocástico.

La tabla 5 presenta los efectos marginales en las probabilidades de la elección. Las variables que miden dinero (W1, W2, W3, B1, B2, C1 y C2) se muestran en $\$ 100 \mathrm{M}$. Como se puede apreciar, las decisiones de los jóvenes dependen fuertemente de los ingresos futuros en los casos de seguir una carrera conducente a un grado profesional y de no continuar estudiando. Así, si el ingreso esperado a los 30 años -cuando la persona se titula de una carrera profesional- aumenta en $\$ 100.000$, la probabilidad de elegirla crece en $1,76 \%$, mientras que si el ingreso esperado a la misma edad en caso de no continuar estudios superiores aumenta en $\$ 100.000$, la probabilidad de seguir esta opción se incrementa en $25 \%$. Los ingresos esperados en caso de titularse de carreras técnicas, en cambio, no son relevantes en las decisiones. Esto es, si los salarios que el mercado laboral otorga a los titulados de carreras técnicas aumentan, no hay un impacto significativo en la probabilidad de estudiar este tipo de carreras. La sensibilidad ante los salarios también varía con la situación económica. La tabla 6 muestra los cambios en la probabilidad de seguir una de las tres opciones al aumentar en \$ 100.000 cada uno de los ingresos esperados asociados 
252 RESTRICCIONES ECONÓMICAS EN ESTUDIOS TÉCNICOS O PROFESIONALES - Luis Hernández, Ricardo Paredes

directamente a la opción. Se desprende de su lectura que los jóvenes con mejor situación económica actual son más sensibles a cambios en los ingresos esperados en el futuro, particularmente en el caso de elegir carreras profesionales o de no continuar estudios superiores, lo que es consistente con las restricciones de liquidez que puedan tener.

Tabla 5: Efectos marginales del modelo de elección

\begin{tabular}{|c|c|c|c|}
\hline & Profesional & Técnico & Trabajo \\
\hline Variable & $\begin{array}{c}\mathrm{dy} / \mathrm{d} x \\
(\mathrm{z})\end{array}$ & $\begin{array}{c}\mathrm{dy} / \mathrm{d} x \\
(z)\end{array}$ & $\begin{array}{l}\mathrm{dy} / \mathrm{d} x \\
(z)\end{array}$ \\
\hline RA & $\begin{array}{r}0,0083883^{*} \\
(35,57) \\
\end{array}$ & $\begin{array}{r}0,009167^{*} \\
(56,61) \\
\end{array}$ & $\begin{array}{r}-0,0175553^{*} \\
(-64,46) \\
\end{array}$ \\
\hline $\mathrm{RA}^{2}$ & $\begin{array}{r}-0,00000431^{*} \\
(-18,90)\end{array}$ & $\begin{array}{r}-0,00000850^{*} \\
(-54,29)\end{array}$ & $\begin{array}{r}0,0000128^{*} \\
(48,38)\end{array}$ \\
\hline Y1 & $\begin{array}{r}0,0176^{*} \\
(27,09)\end{array}$ & $\begin{array}{r}-0,00125^{*} \\
(-2,74)\end{array}$ & $\begin{array}{r}-0,0163^{*} \\
(-21,35)\end{array}$ \\
\hline Y2 & $\begin{array}{l}0,134^{*} \\
(99,88)\end{array}$ & $\begin{array}{r}0,0177^{*} \\
(18,99)\end{array}$ & $\begin{array}{l}-0,152^{*} \\
(-97,76)\end{array}$ \\
\hline Y3 & $\begin{array}{l}-0,240^{*} \\
(-14,10)\end{array}$ & $\begin{array}{r}-0,00973^{*} \\
(-6,85)\end{array}$ & $\begin{array}{r}0,250^{*} \\
(104,95)\end{array}$ \\
\hline $\mathrm{Bl}$ & $\begin{array}{l}-0,400^{*} \\
(-49,91)\end{array}$ & $\begin{array}{l}0,188^{*} \\
(35,46)\end{array}$ & $\begin{array}{l}0,212^{*} \\
(24,21)\end{array}$ \\
\hline B2 & $\begin{array}{r}0,0117^{*} \\
(4,89)\end{array}$ & $\begin{array}{r}-0,0421^{*} \\
(-25,22)\end{array}$ & $\begin{array}{r}0,0305^{*} \\
(11,33)\end{array}$ \\
\hline $\mathrm{Cl}$ & $\begin{array}{r}-0,0596^{*} \\
(-10,89)\end{array}$ & $\begin{array}{r}-0,0607^{*} \\
(-16,37) \\
\end{array}$ & $\begin{array}{l}0,120^{*} \\
(19,79)\end{array}$ \\
\hline $\mathrm{C} 2$ & $\begin{array}{l}0,710^{*} \\
(50,42) \\
\end{array}$ & $\begin{array}{l}-0,139^{*} \\
(-15,09) \\
\end{array}$ & $\begin{array}{l}-0,571^{*} \\
(-37,00) \\
\end{array}$ \\
\hline Q1 & $\begin{array}{r}-0,1299824^{*} \\
(-86,45) \\
\end{array}$ & $\begin{array}{r}-0,0657165^{*} \\
(-73,14) \\
\end{array}$ & $\begin{array}{r}0,195699 * \\
(114,02) \\
\end{array}$ \\
\hline Q2 & $\begin{array}{r}-0,1282023^{*} \\
(-91,83) \\
\end{array}$ & $\begin{array}{r}-0,0416324^{*} \\
(-42,68) \\
\end{array}$ & $\begin{array}{r}0,1698347^{*} \\
(102,25) \\
\end{array}$ \\
\hline Q3 & $\begin{array}{r}-0,0650064^{*} \\
(-43,34) \\
\end{array}$ & $\begin{array}{r}-0,036834^{*} \\
(-39,28) \\
\end{array}$ & $\begin{array}{r}0,1018404^{*} \\
(59,26) \\
\end{array}$ \\
\hline Q4 & $\begin{array}{r}-0,0390107^{*} \\
(-35,87) \\
\end{array}$ & $\begin{array}{r}-0,0280181^{*} \\
(-40,86) \\
\end{array}$ & $\begin{array}{r}0,0670288^{*} \\
(53,14)\end{array}$ \\
\hline $\mathrm{N}$ & 1.874 .764 & & \\
\hline
\end{tabular}

Nota: Los coeficientes significativos al $5 \%$ se indican con * 
Tabla 6: Sensibilidad ante cambios en los ingresos esperados

\begin{tabular}{l|r|r|r|r|r}
\hline Ingreso - Elección & Quintil I & Quintil II & Quintil III & Quintil IV & Quintil V \\
\hline Y1 - Profesional & $1,42 \%$ & $1,43 \%$ & $1,81 \%$ & $1,94 \%$ & $2,10 \%$ \\
\hline Y2 - Técnico & $1,54 \%$ & $2,11 \%$ & $1,66 \%$ & $1,56 \%$ & $1,47 \%$ \\
\hline Y3 - Trabajo & $20,8 \%$ & $21,3 \%$ & $25,6 \%$ & $26,9 \%$ & $28,0 \%$ \\
\hline
\end{tabular}

La probabilidad de estudiar en la educación superior (suma de las probabilidades de las dos opciones de estudios) crece con el rendimiento académico, lo que permite inferir que existe un componente puramente meritocrático en el sistema. Los jóvenes con altos puntajes tienden a preferir carreras profesionales y los con bajos se inclinan a no continuar estudios superiores. La probabilidad de ingresar a una carrera técnica, en cambio, crece hasta alrededor de los 540 puntos, para luego comenzar a disminuir ${ }^{11}$. Tomando a un alumno promedio del tercer quintil, se observa que, hasta los 552 puntos, la alternativa más probable es no continuar estudios, a partir de ese límite ésta es superada por la elección de una carrera profesional con un 44,9\% (figura 1). La probabilidad de ingresar a una carrera técnica tiene forma de campana, con el máximo a los 524 puntos y con una probabilidad de 11,1\%. La forma general que toman las probabilidades respecto del rendimiento académico se mantiene al considerar alumnos promedio de los otros quintiles. Esto indica que la educación superior técnica no tiene un nicho en el cual sea la alternativa más probable, ya que los alumnos de puntajes más bajos no la eligen por las restricciones que enfrentan y los de puntajes medios y altos prefieren ingresar a carreras profesionales. Además, si se igualan los ingresos esperados en la educación profesional y técnica (equiparando hacia arriba o hacia abajo) y se mantiene el control por situación económica y rendimiento académico, la primera sigue siendo la alternativa más probable, lo que muestra que, efectivamente, las carreras superiores técnicas están en desventaja.

11 Esto se obtiene a partir de la regresión (5), calculando $\frac{\text { dELECCIÓN }}{d R A}=\alpha_{1}+2 \alpha_{2} \mathrm{RA}=0$. 
Figura 1: Probabilidades para un alumno promedio del tercer quintil

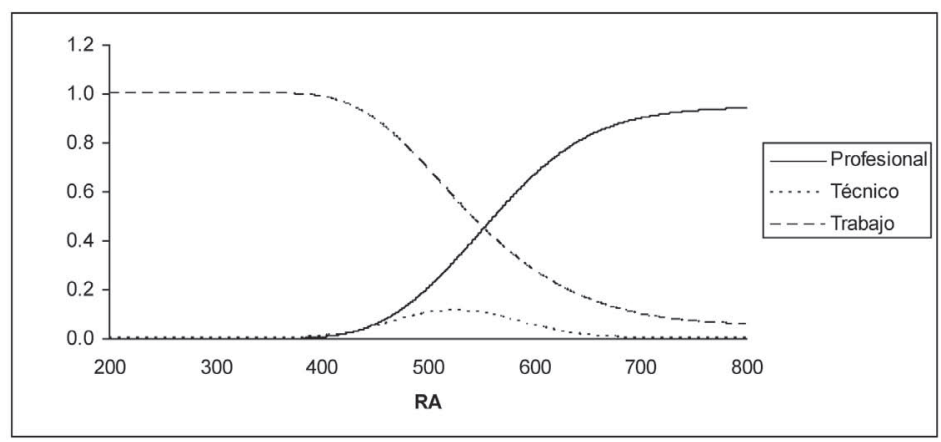

Un mayor índice de becas disminuye la probabilidad de que un individuo elija estudiar una carrera profesional, manteniéndose todo lo demás constante. Esto se entiende por la forma en que son asignadas nacionalmente: solamente las personas que postulan optan a estas becas, quedando fuera muchos jóvenes con alto rendimiento académico que por alguna razón no postularon. Por ejemplo, un egresado de cuarto medio con interés en continuar estudiando, pero que no puede hacerlo por problemas de liquidez, pudiera también ver limitado el acceso por el escaso acceso a la información u otras características socioeconómicas que no están en el modelo. La evidencia muestra que esta situación se repite de tal manera que quienes no estudian tienen más becas y créditos esperados en las dos opciones, por lo que un aumento en la ayuda que una opción de estudios le ofrece hace que la probabilidad de estudiar disminuya ${ }^{12}$. Los resultados también sugieren un monto total insuficiente para resolver las restricciones de liquidez, porque si un joven espera recibir una beca que no le alcance para financiar sus estudios, no postulará aun cuando tenga buen acceso a la información.

La situación económica es clave en la predicción de las probabilidades, evidencia de la presencia de restricciones de liquidez. Si una persona pasa de un quintil al siguiente, la probabilidad de entrar a cualquiera de las dos opciones de estudios aumenta, mientras

12 No hay evidencia de endogeneidad, porque ninguna ayuda estudiantil en una alternativa de estudios depende de las probabilidades de la elección. 
que la probabilidad de no estudiar disminuye. Estas desigualdades en el sistema son mayores en el ingreso a carreras profesionales que a carreras técnicas, debido a que las primeras resultan ser más selectivas precisamente por su mayor valoración. De este modo, una persona que pertenece al primer quintil, en relación con otra del quinto quintil, ceteris paribus, tendrá una probabilidad 13\% menor de ingresar a una carrera profesional y 6,6\% menor de ingresar a una carrera superior técnica (figura 2).

Figura 2: Efectos marginales de cambios en la situación económica

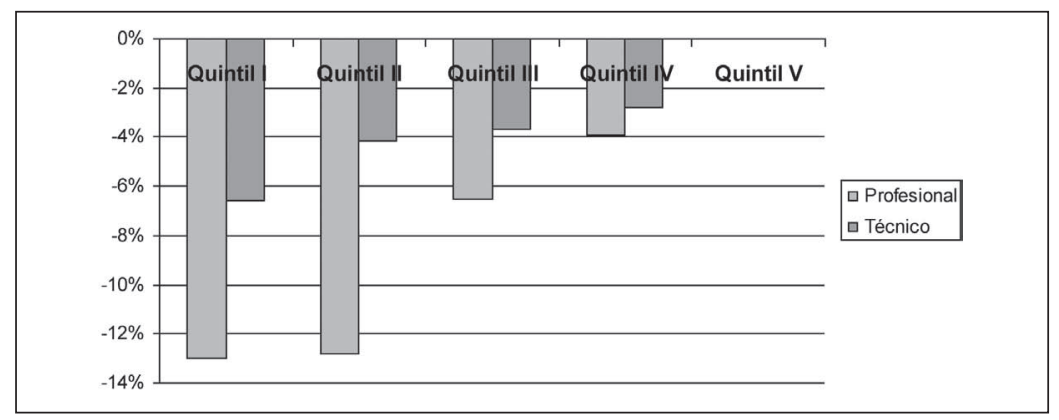

\section{Conclusiones}

Los jóvenes son sensibles a cambios en los ingresos esperados cuando consideran ingresar a una carrera profesional, especialmente los con mejor situación económica. Aquellos de los primeros quintiles, en cambio, tienen la elección relativamente más acotada y muestran una sensibilidad menor. De la misma forma, son sensibles a cambios en los ingresos esperados al evaluar no continuar estudios superiores. Sin embargo, esto no sucede cuando consideran seguir carreras técnicas, debido a que la estructura jerárquica entre las instituciones de educación superior hace que éstas sean la segunda opción que consideren, por lo que quienes las elijan lo harán muy restringidos por la incapacidad de pagar carreras profesionales y por su rendimiento académico.

Consecuentemente, las desigualdades producidas por las restricciones de liquidez son más fuertes en las carreras profesionales 
que reciben a los alumnos con mejor situación económica y capital humano; es esperable, por tanto, que ante una misma educación tengan mejores salarios. Los resultados encontrados sobre ayudas estudiantiles (becas y créditos) muestran que los montos no son suficientes para resolver las restricciones de liquidez y sugieren un problema de eficiencia, por la no postulación de jóvenes que tienen las condiciones para obtener estos beneficios, ya sea por su precaria situación económica o su destacado rendimiento académico. Se requiere un análisis más profundo en este tema, pero se puede concluir que existen problemas de acceso a la información.

La selección de alumnos en la educación superior considera el mérito académico, lo que es muy positivo por ser la principal herramienta de los jóvenes para superar sus restricciones económicas. Los con mejor rendimiento académico tienen prácticamente asegurado su ingreso a una carrera profesional, que es la alternativa más rentable. En este sentido, las carreras profesionales aparecen como el principal vehículo para el movimiento socioeconómico intergeneracional, lo que es coherente con la selectividad, con el hecho de que éstas reciben mayores aportes estatales y con ciertas restricciones al aumento de capacidad.

Por su parte, el acceso a carreras técnicas se da principalmente en alumnos de rendimientos académicos medios, aun cuando, incluso en este rango de puntajes, las carreras profesionales siguen siendo la alternativa más probable. Una asignación de financiamiento público orientada a la educación superior técnica y focalizada en este segmento permitiría no sólo modificar la estructura de la pirámide que relaciona la educación profesional y técnica en Chile, sino que le quitaría a las carreras profesionales el enorme peso que soportan por atender a muchos alumnos que ingresan mal preparados.

Agradecemos los comentarios y sugerencias hechas por Cristian Cox, Elena Puga, José Pedro Undurraga, Marcelo Von Chrismar y Nicolás Majluf. Además, agradecemos al Ministerio de Planificación y Cooperación (MIDEPLAN), al Departamento de Evaluación, Medición y Registros Educacionales (DEMRE) y al Consejo Superior de Educación (CSE) por el acceso a la información. 


\section{Referencias bibliográficas}

Arellano, S. \& Braun, M. (1999) Rentabilidad de la educación formal en Chile. Cuadernos de Economía 107, pp. 685-724.

Becker, G. (1964) Human Capital: A theoretical and empirical análisis, with special reference to education. New York: National Bureau of Economic Research.

Bernasconi, A. (1998) Posibilidades y límites de la información como estrategia para mejorar la calidad de las instituciones de educación superior. Estudios Sociales 95(1), pp. 45-60.

Bernasconi, A. (2006) Donde no somos tigres: Problemas de la formación técnica en Chile en el contexto latinoamericano. Expansiva, Vol. 72.

Bourdieu, P. y Passeron, J.C. (2003) Los herederos. Los estudiantes y la cultura. Buenos Aires: Siglo XXI Editores.

Brunner, J. J. et al. (2005) Guiar el Mercado, Informe sobre la educación superior en Chile: 1980-2003. Santiago: Editorial Universitaria.

Cáceres, C. \& Chávez C. (1995) El programa de becas de arancel universitario Mineduc: Antecedentes para una evaluación. Cuadernos de Economía 96, pp. 165-198.

Dittborn, P. \& Aedo, C. (2005) Oferta, demanda y políticas públicas en educación superior. Calidad en la Educación 22, pp. 53-68.

Galotti, K. \& Mark, M. (1994) How do students structure an important life decision. A short term longitudinal study of the institution decisionmaking process. Research in Higher Educaction 35(5), pp. 589-607.

García de León, M. A. (2005) La Excelencia Científica (Hombres y mujeres en las reales academias). Madrid: Instituto de la Mujer, Ministerio de Trabajo y Asuntos Sociales.

Heckman, J. (1979) Sample selection bias as a specification error. Econometrica 47(1), pp. 153-161.

Hernández, L. (2007) Efectos de las restricciones de liquidez en la decisión de continuar estudios superiores. Tesis de Magíster en Ciencias de la Ingeniería, Pontificia Universidad Católica de Chile, Escuela de Ingeniería.

Hossler, D. et al. (1989) Understanding Student College Choice: Higher Education. Handbook of Theory and Research 5. New York: Agathon Press, pp. 231-288. 
Larrañaga, O. (2006) Costos y beneficios de estudiar en centros de formación técnica: ¿neutralidad de la política pública? Expansiva, Vol. 72.

Leiva, A. (2003) El crédito estudiantil como herramienta de financiamiento universitario. Revista chilena de Administración Pública 2, pp. 141-156.

Leiva, A. (2005) Ejes para la elaboración de una política para la educación superior. Calidad en la Educación 22, pp. 37-52.

Lemaitre, M. J. \& Raczynski, D. (2001) Stakeholders in higher education: How to deal with complexity? Quality Assurance in Higher Education: Quality, Standards and Recognition. $6^{\text {th }}$ Biennal Conference of International Network for Quality Assurance Agencies in Higher Education (INQAAHE), Bangalore, India.

Mincer, J. (1974) Schooling, experience and earnings. New York: Columbia Press University.

Patrinos, H. (1990) The Privatization of Higher Education in Colombia: Effects on Quality and Equity. Higher Education, 20 (2), pp. 161-173.

Palacios, M. (2001) Human capital contracts and human capital options. Characteristic, valuation and implementation. University of Virginia, Darden Graduate School of Business Administration.

PNUD. (2006) Expansión de la Educación Superior en Chile. Boletín informativo del Programa de las Naciones Unidas para el Desarrollo, $\mathrm{N}^{\circ} 10$.

Raczynski, D. \& Canales, A. (2000) Diseño, desarrollo e implementación de un sistema de información pública sobre características de los programas y carreras técnicas, profesionales y de licenciatura ofrecidos por las instituciones de educación superior. Informe final de consultoría. Santiago de Chile: Comisión Nacional de carreras de pregrado y Corporación de Investigaciones Económicas para Latinoamérica (CIEPLAN).

Sapelli, C. (2003) Ecuaciones de Mincer y las tasas de retorno a la educación en Chile: 1990-1998. Documento de Trabajo N²54, Pontificia Universidad Católica de Chile, Instituto de Economía.

Schultz, T. (1960) Capital formation by education. Journal of Political Economy 68(6), pp. 571-583.

Stone, D. (1990) Private higher education in Australia. Higher Education, 20, pp. 143-159.

Tilak, J. (1993) Financing Higher Education in India: principles, practice and policy issues. Higher Education, 26, pp. 43-47. 


\section{ANEXOS}

\section{Anexo A}

Tabla 7: Detalle de los efectos marginales sobre el rendimiento académico

\begin{tabular}{|c|c|c|}
\hline Var & Coef & (z) \\
\hline SEXO & $11,9^{*}$ & $(26,6)$ \\
\hline CPP & $39,4^{*}$ & $(54,8)$ \\
\hline $\mathrm{CMU}$ & $-13,9^{*}$ & $(-27,2)$ \\
\hline EDAD & $-0,1^{*}$ & $(-2,0)$ \\
\hline $\mathrm{TF}$ & $-2,6^{*}$ & $(-20,2)$ \\
\hline R2 & $-4,7^{*}$ & $(-2,8)$ \\
\hline R3 & $-5,3^{*}$ & $(-2,6)$ \\
\hline $\mathrm{R} 4$ & $4,5^{*}$ & $(2,7)$ \\
\hline R5 & $10,4^{*}$ & $(7,5)$ \\
\hline R6 & $15,1^{*}$ & $(9,4)$ \\
\hline R7 & $28,6^{*}$ & $(17,9)$ \\
\hline $\mathrm{R} 8$ & $18,8^{*}$ & $(13,6)$ \\
\hline R9 & $4,3^{*}$ & $(2,7)$ \\
\hline R10 & $12,2^{*}$ & $(7,7)$ \\
\hline R11 & $28,8^{*}$ & $(8,8)$ \\
\hline R12 & $11,7^{*}$ & $(4,9)$ \\
\hline R13 & $9,6^{*}$ & $(7,5)$ \\
\hline $\mathrm{PB}$ & $-7,5^{*}$ & $(-7,8)$ \\
\hline PM & $6,4^{*}$ & $(7,4)$ \\
\hline PTI & $27,8^{*}$ & $(12,4)$ \\
\hline PTC & $32,3^{*}$ & $(25,4)$ \\
\hline PUI & $37,3^{*}$ & $(30,9)$ \\
\hline PUC & $50,7^{*}$ & $(48,4)$ \\
\hline $\mathrm{PO}$ & $22,5^{*}$ & $(14,2)$ \\
\hline $\mathrm{MB}$ & $3,4^{*}$ & $(2,2)$ \\
\hline MM & $20,5^{*}$ & $(13,7)$ \\
\hline MTI & $37,1^{*}$ & $(14,7)$ \\
\hline MTC & $47,0^{*}$ & $(27,3)$ \\
\hline MUI & $50,5^{*}$ & $(28,0)$ \\
\hline MUC & $61,3^{*}$ & $(37,7)$ \\
\hline $\mathrm{MO}$ & $21,4^{*}$ & $(9,7)$ \\
\hline Constante & $466,4^{*}$ & $(223,5)$ \\
\hline $\mathrm{N}$ & 178.652 & \\
\hline
\end{tabular}

Nota: Los coeficientes significativos al $5 \%$ se indican con *.

SEXO es una dummy con valor 1 si es hombre; CPP y CMU son dummies para la persona que asistió a un colegio particular privado 
y municipal, respectivamente (el colegio privado subvencionado es base); EDAD es la edad de la persona en años; TF es el número de integrantes de la familia; Rj es una dummy para la región k (primera región es base); PB, PM, PTI, PTC, PUI, PUC y PO son dummies para cada nivel educacional del padre, siendo de básica, de media, técnico incompleto, técnico completo, universitario incompleto, universitario completo y otro, respectivamente (sin estudios es base); MB, MM, MTI, MTC, MUI, MUC y MO se definen análogamente, pero para el nivel educacional de la madre.

\section{Anexo B}

Tabla 8: Detalle de los efectos marginales en la probabilidad de venir de cada tipo de colegio

\begin{tabular}{l|c|c|c|c|c|c}
\hline \multicolumn{3}{c|}{ Municipal } & \multicolumn{2}{l|}{ Part subv. } & \multicolumn{2}{l}{ Part. pagado } \\
\hline Var & dy/dx & $(z)$ & dy/dx & $(z)$ & dy/dx & $(z)$ \\
\hline SEXO & $0,009^{*}$ & $(16,0)$ & $-0,006^{*}$ & $(-11,8)$ & $-0,003^{*}$ & $(-11,5)$ \\
\hline D1 & $0,394^{*}$ & $(483,5)$ & $-0,318^{*}$ & $(-396,9)$ & $-0,075^{*}$ & $(-390,1)$ \\
\hline D2 & $0,409^{*}$ & $(529,0)$ & $-0,333^{*}$ & $(-437,5)$ & $-0,076^{*}$ & $(-393,7)$ \\
\hline D3 & $0,361^{*}$ & $(406,4)$ & $-0,285^{*}$ & $(-325,6)$ & $-0,076^{*}$ & $(-390,1)$ \\
\hline D4 & $0,336^{*}$ & $(357,2)$ & $-0,262^{*}$ & $(-281,9)$ & $-0,075^{*}$ & $(-383,1)$ \\
\hline D5 & $0,312^{*}$ & $(314,0)$ & $-0,237^{*}$ & $(-241,8)$ & $-0,075^{*}$ & $(-385,5)$ \\
\hline D6 & $0,285^{*}$ & $(272,4)$ & $-0,212^{*}$ & $(-205,6)$ & $-0,072^{*}$ & $(-376,0)$ \\
\hline D7 & $0,223^{*}$ & $(193,1)$ & $-0,153^{*}$ & $(-133,8)$ & $-0,070^{*}$ & $(-370,2)$ \\
\hline D8 & $0,159^{*}$ & $(127,8)$ & $-0,093^{*}$ & $(-75,8)$ & $-0,066^{*}$ & $(-360,3)$ \\
\hline D9 & $0,087^{*}$ & $(65,3)$ & $-0,032^{*}$ & $(-24,7)$ & $-0,055^{*}$ & $(-330,8)$ \\
\hline R2 & $0,197^{*}$ & $(104,4)$ & $-0,202^{*}$ & $(-121,0)$ & $0,005^{*}$ & $(4,4)$ \\
\hline R3 & $0,176^{*}$ & $(76,6)$ & $-0,171^{*}$ & $(-81,2)$ & $-0,005^{*}$ & $(-4,5)$ \\
\hline R4 & $0,014^{*}$ & $(6,9)$ & $-0,046^{*}$ & $(-23,6)$ & $0,031^{*}$ & $(23,0)$ \\
\hline R5 & $-0,077^{*}$ & $(-42,2)$ & $0,007^{*}$ & $(4,1)$ & $0,070^{*}$ & $(47,9)$ \\
\hline R6 & $0,060^{*}$ & $(28,5)$ & $-0,091^{*}$ & $(-48,1)$ & $0,032^{*}$ & $(22,9)$ \\
\hline R7 & $0,146^{*}$ & $(80,9)$ & $-0,153^{*}$ & $(-94,0)$ & $0,007^{*}$ & $(7,0)$ \\
\hline R8 & $0,069^{*}$ & $(39,2)$ & $-0,096^{*}$ & $(-60,2)$ & $0,028^{*}$ & $(25,2)$ \\
\hline R9 & $-0,107^{*}$ & $(-56,5)$ & $0,091^{*}$ & $(48,2)$ & $0,016^{*}$ & $(14,2)$ \\
\hline R10 & $0,098^{*}$ & $(54,6)$ & $-0,116^{*}$ & $(-70,8)$ & $0,018^{*}$ & $(16,6)$ \\
\hline R11 & $0,010^{*}$ & $(2,6)$ & $0,037^{*}$ & $(9,5)$ & $-0,048^{*}$ & $(-250,6)$ \\
\hline R12 & $0,145^{*}$ & $(46,7)$ & $-0,147^{*}$ & $(-51,3)$ & 0,001 & $(0,9)$ \\
\hline R13 & $-0,115^{*}$ & $(-71,1)$ & $0,058^{*}$ & $(37,2)$ & $0,057^{*}$ & $(62,1)$ \\
\hline N & 3.809 .754 & & \multicolumn{5}{|c}{} & \\
\hline
\end{tabular}

Nota: Los coeficientes significativos al $5 \%$ se indican con *.

SEXO es una dummy con valor 1 si es hombre; Dj es una dummy para el decil j (decil 10 es base); Rj es una dummy para la región j (región 1 es base). 


\section{Anexo C}

Tabla 9: Detalle de los efectos del modelo de Heckman de ingresos

\begin{tabular}{l|c|c|c|c|c|c}
\hline \multicolumn{3}{|c|}{ Profesional } & \multicolumn{2}{c|}{ Técnico } & \multicolumn{2}{c}{ Trabajo } \\
\hline Var & Coef & $(z)$ & Coef & $(z)$ & Coef & $(z)$ \\
\hline EDAD & $0,08^{*}$ & $(103,05)$ & $0,05^{*}$ & $(58,1)$ & $0,07^{*}$ & $(129,2)$ \\
\hline EDAD & $-0,00^{*}$ & $(-98,42)$ & $0,00^{*}$ & $(-44,6)$ & $0,00^{*}$ & $(-120,7)$ \\
\hline SEXO & $0,67^{*}$ & $(116,94)$ & $0,50^{*}$ & $(47,7)$ & $0,58^{*}$ & $(117,2)$ \\
\hline R2 & $0,07^{*}$ & $(6,95)$ & $-0,05^{*}$ & $(-5,03)$ & $0,21^{*}$ & $(42,17)$ \\
\hline R3 & $0,25^{*}$ & $(18,76)$ & 0,01 & $(0,91)$ & $-0,08^{*}$ & $(-13,17)$ \\
\hline R4 & 0,00 & $(0,12)$ & $-0,20^{*}$ & $(-18,37)$ & $-0,11^{*}$ & $(-21,63)$ \\
\hline R5 & $-0,15^{*}$ & $(-17,92)$ & $-0,21^{*}$ & $(-24,13)$ & $-0,05^{*}$ & $(-13,74)$ \\
\hline R6 & $-0,06^{*}$ & $(-5,84)$ & $-0,18^{*}$ & $(-17,16)$ & $0,05^{*}$ & $(9,63)$ \\
\hline R7 & 0,01 & $(0,85)$ & $-0,29^{*}$ & $(-26,9)$ & $-0,06^{*}$ & $(-12,74)$ \\
\hline R8 & $0,05^{*}$ & $(6,8)$ & $-0,25^{*}$ & $(-27,72)$ & $-0,10^{*}$ & $(-24,39)$ \\
\hline R9 & $-0,13^{*}$ & $(-14,89)$ & 0,01 & $(0,83)$ & $-0,12^{*}$ & $(-25,55)$ \\
\hline R10 & $0,20^{*}$ & $(23,6)$ & $-0,18^{*}$ & $(-18,15)$ & $0,09^{*}$ & $(20,62)$ \\
\hline R11 & $0,46^{*}$ & $(26,21)$ & $0,28^{*}$ & $(12,45)$ & $0,39^{*}$ & $(35,1)$ \\
\hline R12 & $0,19^{*}$ & $(11,09)$ & $0,05^{*}$ & $(3,22)$ & $0,48^{*}$ & $(69,79)$ \\
\hline R13 & $0,36^{*}$ & $(50,56)$ & $-0,01$ & $(-1,06)$ & $0,22^{*}$ & $(58,45)$ \\
\hline ECB & $-0,77^{*}$ & $(-71,21)$ & $-0,46^{*}$ & $(-46,03)$ & $-0,34^{*}$ & $(-96,77)$ \\
\hline ECM & $-0,22^{*}$ & $(-55,94)$ & $-0,18^{*}$ & $(-29,61)$ & $-0,04^{*}$ & $(-12,04)$ \\
\hline ECTI & $-0,10^{*}$ & $(-13,44)$ & $-0,16^{*}$ & $(-17,98)$ & $-0,09^{*}$ & $(-23,76)$ \\
\hline ECTC & $-0,06^{*}$ & $(-14,33)$ & $-0,01$ & $(-1,32)$ & $0,01^{*}$ & $(3,43)$ \\
\hline ECUI & $0,25^{*}$ & $(52,29)$ & $-0,05^{*}$ & $(-5,26)$ & $0,03^{*}$ & $(6,17)$ \\
\hline ECUC & $0,02^{*}$ & $(2,9)$ & $0,04^{*}$ & $(4,01)$ & $0,07^{*}$ & $(14,43)$ \\
\hline ECO & $-0,33^{*}$ & $(-66,47)$ & $-0,21^{*}$ & $(-26,95)$ & $-0,17^{*}$ & $(-48,62)$ \\
\hline Constante & $10,44^{*}$ & $(394,10)$ & $11,20^{*}$ & $(404,3)$ & $10,00^{*}$ & $(564,42)$ \\
\hline$\lambda$ & $0,83^{*}$ & $(25,64)$ & $-0,10^{*}$ & $(-3,05)$ & $0,13^{*}$ & $(12,61)$ \\
\hline N & 635.706 & & 377.091 & & 1.946 .254 & \\
\hline
\end{tabular}

Nota: Los coeficientes significativos al 5\% se indican con *

$E D A D$ es la edad de la persona en años; Rj es una dummy para la región j (región 1 es base); ECB, ECM, ECTI, ECTC, ECUI, ECUC y ECO son dummies para cada nivel educacional del cónyuge, siendo de básica, de media, técnico incompleto, técnico completo, universitario incompleto, universitario completo y otro, respectivamente (sin estudios es base); y $\lambda$ es el inverso de la razón de Mills.

Recibido:15 de agosto de 2007

Aceptado: 27 de septiembre de 2007 